\title{
Quality Nursing Education in Unprecedented Times - Teaching and Learning for the Unexpected I La qualité de la formation en sciences infirmières en cette période sans précédent - apprendre et enseigner pour l'inattendu
}

Florence Myrick

amyrick@ualberta.ca

Jacinthe I. Pepin

Université de Montréal, jacinthe.pepin@umontreal.ca

Follow this and additional works at: https://qane-afı.casn.ca/journal

Part of the Higher Education Commons, and the Nursing Commons

\section{Recommended Citation}

Myrick, Florence and Pepin, Jacinthe I. (2020) "Quality Nursing Education in Unprecedented Times - Teaching and Learning for the Unexpected I La qualité de la formation en sciences infirmières en cette période sans précédent apprendre et enseigner pour l'inattendu," Quality Advancement in Nursing Education - Avancées en formation infirmière: Vol. 6: Iss. 1, Article 1.

DOI: https://doi.org/10.17483/2368-6669.1242

This Editorial is brought to you for free and open access by Quality Advancement in Nursing Education - Avancées en formation infirmière. It has been accepted for inclusion in Quality Advancement in Nursing Education - Avancées en formation infirmière by an authorized editor of Quality Advancement in Nursing Education - Avancées en formation infirmière. 


\section{Quality Nursing Education in Unprecedented Times - Teaching and Learning for the Unexpected}

Who could have anticipated the circumstances in which we find ourselves today? We refer to the emergence of the coronavirus (COVID-19) pandemic and its impact globally, not only on population health, the health care system but equally on nursing education. We are thus compelled to ponder and ask ourselves as educators and academic managers, how do we continue to provide quality nursing education within the context of these unprecedented and challenging times? At the same time, how do we ensure that nurses for the future develop as health professionals who are adequately prepared to contend with such uncertainty?

Fortunately, to date, we have already established partial or complete courses and programs, which are offered online. In addition, we have created virtual clinical simulations. Moreover, each institution of higher education provides a digital learning environment allowing students immediate access to learning materials, activities, and assessments. Other team and group communication tools are now made readily accessible for distant teaching. This process affords students, teachers and health care professionals the opportunity to obtain or deliver grades with the support of this technology which, in prior decades, may not have been so readily available. In this time of social/physical distancing, we need to capitalize on our strengths while acknowledging those areas which require more creativity, collective ingenuity and consideration such as quality clinical placements. It is also an opportunity to advance innovative teaching and learning strategies that a) align with curricular values, b) are student-centred, c) promote active and reflective/reflexive learning, and d) provide constructive feedback.

Among quality safeguards, we particularly want to highlight research and accreditation. Nursing educational research is essential for the exploration and evaluation of potential approaches to expand cutting-edge competencies that will enable nurses of the future to respond knowledgeably and indeed competently to unanticipated or unexpected health care events. Equally essential is evaluation research especially when implementing new strategies with limited resources. And let us not forget the accreditation program which is fundamental to quality nursing education which is also impacted by these global events. Invariably, changes in some key elements or in the delivery of the accreditation process may also be precipitated by such major events. Within this context, communication technologies could be of invaluable assistance.

Finally, we would be remiss in the midst of a time such as this were we not to acknowledge the incredible work of our fellow nurses in the health care system who daily work diligently to keep the public safe and healthy. Not enough acknowledgement can be given to their unwavering commitment.

Exceptionally, this current issue does not include an Interview. This section will resume in the Fall 2020 Issue.

Florence Myrick and Jacinthe Pepin, Co-Editors-in-Chief 


\section{La qualité de la formation en sciences infirmières en cette période sans précédent - apprendre et enseigner pour l'inattendu}

Qui aurait pu prévoir les circonstances dans lesquelles nous nous trouvons aujourd'hui? Nous parlons de la pandémie du nouveau coronavirus (COVID-19) et de son impact, à l'échelle mondiale, sur la santé des populations, sur les systèmes de santé, et certainement sur la formation en sciences infirmières. Ce contexte particulier et rempli de défis force les formateurs et les gestionnaires académiques à se demander comment continuer à dispenser une formation de qualité? En même temps, comment veiller à ce que les infirmières de demain se développent en tant que professionnelles de la santé qui sont suffisamment préparées pour faire face à de telles incertitudes?

Heureusement, à ce jour, nous avons déjà créé des cours et des programmes, entièrement ou partiellement offerts en ligne. Aussi, nous avons développé des simulations cliniques virtuelles. De plus, chaque milieu d'enseignement supérieur fournit un environnement numérique d'apprentissage qui permet aux étudiants d'accéder en tout temps au matériel d'apprentissage, aux différentes activités et aux divers modes d'évaluation. D'autres outils de communication en équipe ou en grand groupe ont été rapidement rendus disponibles pour faciliter l'offre de cours à distance. Ce processus permet ainsi aux étudiants, aux enseignants et aux professionnels de la santé d'obtenir ou de décerner des grades, avec le soutien de la technologie qui, au cours des décennies précédentes, n'était peut-être pas si facilement disponible. En cette période de distanciation sociale / physique, nous devons capitaliser sur nos forces tout en reconnaissant les zones qui nécessitent plus de créativité, d'ingénuité collective et de considération, comme l'offre de stages cliniques de qualité. C'est aussi l'occasion de faire progresser des stratégies d'enseignement et d'apprentissage innovantes qui: a) s'alignent aux valeurs et finalités du curriculum; b) sont centrées sur l'apprenant; c) promeuvent un apprentissage actif et réflexif; et d) fournissent une rétroaction constructive.

Parmi les balises de qualité, nous voulons particulièrement souligner la recherche et l'accréditation. La recherche en formation en sciences infirmières est essentielle à l'exploration et à l'évaluation d'approches qui ont le potentiel de soutenir le développement de compétences de pointe qui permettront aux infirmières de demain de répondre de manière éclairée et efficace aux situations de soins de santé imprévues ou subites. La recherche évaluative est également essentielle lors de la mise en œuvre de nouvelles stratégies en contexte de ressources limitées. De plus, le programme d'accréditation, assurant la qualité des programmes de formation infirmière, est aussi touché par de tels événements mondiaux. Inévitablement, des changements dans la formulation de certains éléments clés ou dans la mise en œuvre du processus d'accréditation seront précipités par de tels événements majeurs. Dans ce contexte, les technologies de la communication pourraient être d'une aide inestimable.

Enfin, au cœur d'une période comme celle-ci, nous serions négligents de ne pas reconnaitre l'excellent travail de nos collègues infirmières au sein du système de santé qui travaillent quotidiennement avec diligence pour assurer la sécurité et la santé du public. Nous ne pourrions reconnaître suffisamment leur engagement indéfectible.

Exceptionnellement le présent numéro ne comporte pas d'entrevue. Cette section sera reprise à l'automne 2020.

Florence Myrick et Jacinthe Pepin, Corédactrices en chef 